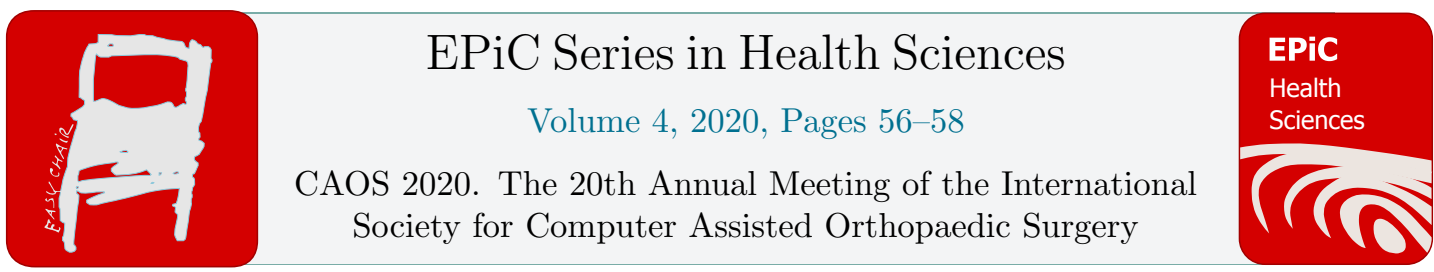

\title{
A Detailed Analysis of Time taken in Robotic Surgery for Total Knee Replacement Arthroplasty and Comparison with Computer Assisted Navigation in Total Knee Replacement Arthroplasty
}

\author{
Kamal Deep, Frederic Picard \\ Nuffield Hospital and Golden Jubilee National Hospital Glasgow UK \\ mrkdeep@gmail.com
}

\begin{abstract}
The accuracy of implantation using computer navigation and robotic total knee replacement (TKR) has been proven. Time taken during surgery has been a factor for surgeons for not using the technology. Aim of this study was to analyse time taken in different steps and identify which part needs improvement. Robotic time was compared to computer navigation. Methods: 15TKR were performed with MAKO robot. Software for the ligament balancing was used. All had CT scan preoperatively. Time of different surgical steps was recorded. Time for computer navigation was recorded too. After joint exposure, trackers and verification pins for tibia and femur were inserted. Femoral registration matching started at 10.8 minutes (SD3.3 Range7-20). It took 3.2 minutes to match femoral anatomy to CT scan. Tibial registration done at 14.1minutes (SD3 Range10-23). Once matching was accepted to required accuracy, tibial cut was made at 22.2 minutes (SD4.4 Range 15-30). Next the soft tissues were assessed with tensioner. It took 6.3 minutes (SD 5.6). Final femoral preparation done at 35.7 minutes (SD 5.6 Range25-45). Trial performed at 52 minutes (SD7.3 Range42-63). Implants were cemented at 63.4 minutes (SD8 Range50-72). Wound closed at 77.6 minutes (SD9.5 Range65-97). The computer navigated TKR surgery took 70 minutes on an average. Compared to navigation, robotic technique took approximately 7 minutes longer, not significantly different. This could be due to learning curve of the surgical and theatre team. Improvement is required in different steps. The familiarity of staff will increase the efficiency. Registration matching took 11.4 minutes. Femoral preparation took 17 minutes. These steps could be streamlined.
\end{abstract}

Introduction: The accuracy of implantation using computer assisted navigation (1-4) and robotic total knee replacement arthroplasty (TKR) has been proven and accepted (5-7). The time taken for the use of technology during surgery, has been one of the major factors quoted by many surgeons 
for not using and accepting the technology in the procedure. Aim: Aim of the present study was to analyse the time taken during different steps of the procedure of TKR, one by one and identify which part needs further improvement. Secondary aim was to compare the time take for use of robotic technology compared to the computer navigation technology. Methods: 15 total joint replacements were carried out with MAKO robotic system. We used the software for the ligament and gap balancing. Same prosthesis was used for all the cases which was cemented on both tibia and femur. All patients had a CT scan preoperatively to plan the surgery. An anterior incision and medial parapatellar approach was used along with tourniquet for all the patients. The times of different surgical steps were noted by a person who did not leave the theatre during the procedure and paid attention to the different steps involved to record accurately. We also looked at the total time taken for using a imageless computer navigation technique for doing the TKR. Results and analysis: After the incision was made, and joint exposed the trackers for the tibia and femur were inserted, tightened and verification pins inserted. It took total about 10 minutes for these steps. Femoral registration matching was then started at 10.8 minutes (SD3.3 Range 7-20) post incision. It took about 3.2 minutes on an average to match the femoral anatomy to the pre op CT scan anatomy. Tibial registration was then started at 14.1 minutes (SD3 Range 10-23) post incision. Once matching was accepted to required accuracy, tibial cut was made. Tibial cut was started at 22.2 minutes (SD4.4 Range 15-30) post incision. Osteophytes were then taken out and the meniscal remnants removed. Next step was then taken to assess the soft tissues with a tensioner and plan the femoral cuts. It took on an average about 6.3 minutes (SD 5.6). Final bone preparation was started for femur at 35.7 minutes (SD 5.6 Range 25-45) post incision. Trial of the prosthesis was done at 52 minutes (SD 7.3 Range 42- 63) after start of the procedure. Cementing of the definite prosthesis was performed at 63.4 minutes (SD 8 Range 50-72). The surgical wound was closed at 77.6 minutes post incision (SD9.5 Range 65-97). The computer navigated TKR surgery took 70 minutes on an average. Compared to navigated knee replacements the robotic technique took approximately 7 minutes longer on an average. This could be due to surgeon having done huge volumes of navigated surgeries while the robotic technique was new. The surgical scrub team was also new which could explain the difference in the navigated vs robotic techniques. While looking at different stages of the procedure there is room for improvement in various steps. The familiarity of all the staff involved with the system will undoubtedly increase the speed and efficiency of the procedure at all the steps. There were two main steps, which took time and could be modified. One is registration matching time for the bone anatomy matching procedure including femoral and tibial matching. There was a difference of an average 11.4 minutes after the tracker insertion to the tibial cut. This step could be quicker if the registration matching time could be reduced for both femur and tibia. It took about 17 minutes on an average for preparation of the femur. This included making adjustments, clearing the soft tissues and making the bone cuts. The saw needs a change of handle in between the cuts once. Some time is spent in making sure the soft tissues are out of the way and due to directions of trackers and camera, which can be a bit awkward with surgeon retracting and doing the cuts at same time. This process could be more streamlined and made quicker and simpler. At final conclusion the robotic procedure of TKR does not take much longer than the navigated techniques. There are various parts of the steps, especially registration of anatomy, which can be modified to make it faster. It is up to individual surgeons how much time they wish to spend for the accuracy of the implantation. Hopefully with future developments the procedure can be made faster than conventional surgery.

\section{References}

1. Deep K, Shankar S \& Mahendra A (2017) Computer assisted navigation in total knee and hip arthroplasty. SICOT J, 3:50. 
2. Picard F, Deep K, Jenny JY (2016) Current state of the art in total knee arthroplasty computer navigation. Knee Surgery, Sport Traumatol Arthrosc 24(11), 3565-3574.

3. Hetaimish BM, Khan MM, Simunovic N, Al-Harbi HH, Bhandari M, Zalzal PK (2012) Metaanalysis of navigation vs conventional total knee arthroplasty. J Arthroplasty 27(6), 1177-1182.

4. Alcelik IA, Blomfield MI, Diana G, Gibbon AJ, Carrington N, Burr S (2016) A comparison of short-term outcomes of minimally invasive computer-assisted vs minimally invasive conventional instrumentation for primary total knee arthro- plasty: a systematic review and meta-analysis. $\mathrm{J}$ Arthroplasty 31(2), 410-418.

5. Lonner JH, Fillingham YA, Pros FYA. Pros and cons: a balanced view of robotics in knee arthroplasty. J Arthroplasty 2018;33:2007-13.

6. Jacofsky DJ, Allen M. Robotics in arthroplasty: a comprehensive review. J Arthroplasty 2016;31:2353-63.

7. Mannan A, Vun J, Lodge C, et al. Increased precision of coronal plane outcomes in roboticassisted total knee arthroplasty: A systematic review and meta-analysis. Surgeon 2018;16:237-44 